\title{
Éditorial:
}

\section{Les sciences humaines reliées au vieillissement}

Les sciences humaines en gérontologie constituent un sujet substantiel et diversifié. Il existe actuellement plusieurs ouvrages qui essaient de fournir une représentation de diverses disciplines intéressées au vieillissement. Les auteurs de ces textes appartiennent à des domaines tels l'histoire, la littérature, la philosophie, la théologie, les beaux-arts, l'anthropologie, etc. Nous attirons l'attention de nos lecteurs sur deux livres parus récemment qui constituent d'excellents outils pour l'enseignement des sciences humaines en gérontologie.

Le premier ouvrage s'intitule The Handbook of the Humanities and Aging (de Cole, Van Tassel, \& Kastenbaum, 1992), et le second Voices and Vision of Aging (de Cole, Achenbaum, Jakobi, \& Kastenbaum, 1993). Ces deux livres non seulement constituent une initiation à l'enseignement des sciences humaines en gérontologie, mais ils fournissent d'autres références relatives à des champs d'intérêt spécifiques. En premier lieu, il y a une critique importante que je me dois de formuler envers ces deux ouvrages : tous les auteurs sont américains. Il n'y a rien de mal à ça, sauf qu'il faut se rappeler qu'ils n'exposent donc pas toutes les notions des sciences humaines en gérontologie. L'espace me manque pour dresser une liste de tous les chercheurs dans ce domaine, mais je peux affirmer que d'excellents travaux émanent actuellement de l'Europe, de la Scandinavie et du Canada. Selon moi, en cette ère que de nombreux auteurs qualifient de " post-contemporaine, " nous avons besoin d'une voix et d'une vision aussi fortes que possible.

En dépit de cette critique, la rédaction d'un ouvrage comme ceux susmentionnés ou la publication d'un numéro spécial portant sur ce sujet vaste et diversifié représente un défi de taille. Dans le cas du présent numéro, la sélection des articles s'est inspirée de la formule de l'émission de la Revue. En effet, après avoir procédé à une présélection, nous avons lancé un appel pour recevoir d'autres textes. Il est à noter que tous les textes ont été soumis au procédé de révision usuel. Je tiens à rappeler que ce numéro spécial comprend plusieurs textes portant sur des aspects particuliers des sciences humaines en gérontologie. L'importance et la valeur de ce numéro résultent de la contribution spécifique de chaque article à notre compréhension du vieillissement.

Cela étant dit, il y a quand même une contribution généralisable qui peut être définie. Chaque article, à sa manière, se penche sur la façon dont nous, à titre de chercheurs, de décideurs, de praticiens et de particuliers, prenons des décisions ayant trait au vieillissement dans nos champs de compétence respectifs. Les sciences humaines explorent la dimension herméneutique du savoir en remettant en question les valeurs, hypothèses et métaphores avec 
lesquelles nous travaillons, de façon implicite ou explicite (Kenyon, Birren, \& Schroots, 1991). Grâce à cette dimension d'autoréflexion, les sciences humaines en gérontologie aident globalement à ce que notre compréhension du vieillissement reflète tous les aspects de la nature humaine, c'est-à-dire les volets physique, psychologique, social et spirito-existentiel. De plus, elles procurent à notre pratique une dimension éthique. En d'autres termes, elles humanisent nos systèmes en nous fournissant une vaste tribune qui englobe ou complète notre raisonnement politique, économique ou autre (Kenyon \& Davidson, 1993).

Le présent numéro se compose de six articles. Les quatre premiers portent sur le vieillissement individuel ou personnel, tandis que les deux autres traitent des aspects socio-structurels du vieillissement qui, en fin de compte, ont des répercussions sur le vieillissement individuel.

Les textes de Harrison, Boetzkes, et Checkland et Silberfeld abordent la constellation cruciale des concepts d'autonomie, de compétence et d'identité. C'est sur ces notions que les décisions d'intervention sont prises dans le domaine du vieillissement. Le dialogue dans ce contexte touche principalement les disciplines de la médecine, de l'éthique et du droit. Cependant, outre ces disciplines, ces trois articles proposent davantage qu'une discussion philosophique et théorique sur la signification de certains termes et traitent plutôt des manières dont nous pourrions appliquer plus efficacement les notions de compétence et d'autonomie dans la réalité quotidienne. Ces articles se caractérisent par leur aspect ontologique, en ce sens qu'ils nous exhortent à reconnaître le volet autocréateur et introspectif de la nature humaine. Pour Harrison et Boetzkes, une façon très efficace d'atteindre cet objectif consiste à mettre l'accent sur des études de cas et sur la dimension narrative de la vie humaine. Selon Boetzkes, cette approche aidera à minimiser les contraintes externes et les risques de coercition relativement à l'élaboration de directives en matière de soins de la santé spécialisés.

Dans le texte de Harrison, l'accent mis sur le récit nous rappelle qu'une personne atteinte de maladie mentale demeure un être humain doté d'une valeur morale et ayant quelque chose à dire. Cette perception nous encourage à considérer non seulement les pertes, mais également les forces que cette personne possède toujours. Bref, cette situation extrêmement idiosyncrasique et dynamique nécessite des interventions personnalisées qui sont accessibles par l'entremise d'études de cas. (Pour un traitement plus approfondi des questions théoriques et pratiques relatives au vieillissement et des études de cas, consultez l'ouvrage de Birren, Kenyon, Ruth, Schroots, \& Svensson qui paraîtra sous peu.)

Checkland et Silberfeld s'intéressent également à l'orientation contextuelle des interventions et mettent l'accent sur la distinction entre l'autonomie et la compétence. Ils proposent comme principe de base que des paramètres clairement établis pour la notion de compétence nous seront plus utiles que de vagues tentatives de définir des concepts tels que l'autonomie. Bien qu'ils ne traitent pas de récits et d'études de cas, ils préconi- 
sent une évaluation dynamique et idiosyncrasique de la notion de compétence fondée sur des facteurs comme des " modèles d'authenticité " changeants et, j'ajouterais, des sources de signification. Un aspect intéressant de tous ces documents est le fait que la discussion s'applique autant aux aînés compétents qu'à ceux qui ne le sont pas. L'incompétence n'élimine pas l'identité.

L'article de Manning nous donne un aperçu des aspects spirito-existentiels du vieillissement. Pour Manning, la littérature peut contribuer à notre compréhension du vieillissement par l'entremise de "fictions imaginatives et d'évocations lyriques d'expériences ». Ces produits de l'imagination semblent s'appliquer à des vies et des situations réelles et, par conséquent, fournissent une autre source de savoir pour la recherche et la pratique en gérontologie. Plus particulièrement, il nous conseille vivement de regarder au-delà de la fragilité physique et d'envisager la possibilité que des aînés désoeuvrés peuvent transcender cette fragilité de façon très significatives. Dans ce sens, on pourrait dire qu'une personne âgée peut avoir l'air frêle tout en étant très compétente.

Dans les cinquième et sixième textes, l'accent est moins mis sur le vieillissement individuel en tant que tel, et davantage sur les relations structuro-systématiques qui définissent également l'expérience du vieillissement. Dans le cinquième article, Clark souligne qu'en bout de ligne, les hypothèses et les valeurs qui guident les procédés et les décisions ayant trait aux politiques publiques influencent de façon significative le vieillissement individuel, notamment le degré de vieillissement reflété dans les diverses politiques et les répercussions de celles-ci sur la qualité de vie. En outre, les hypothèses sous-tendant les politiques publiques influencent également la façon dont les gens intériorisent leur propre vieillissement, par exemple à l'intérieur d'un problème ou d'une célébration. Au moyen d'une comparaison entre la situation actuelle au Canada et celle qui prévaut aux États-Unis, Clark prétend qu'il faut, particulièrement aux États-Unis, instaurer une "éthique publique, " dans le cadre de laquelle le vieillissement et des concepts connexes tels que l'autonomie seront soumis à un dialogue de nature communautaire. De cette façon, un équilibre peut être atteint entre les responsabilités et droits individuels d'une part, et la responsabilité de la société envers l'autonomie dans un pays civilisé d'autre part. Selon Clark, les aînés doivent véritablement avoir les outils requis pour formuler les décisions et solutions touchant les politiques publiques.

Dans le dernier article, Snell aborde, au moyen d'une analyse historique, un autre aspect structural du vieillissement. Il cite un exemple de la façon dont l'inégalité des sexes a été systématiquement favorisée par les politiques régissant les pensions de vieillesse au Canada. Il soutient que les croyances fondamentales à propos du mariage ont eu la préséance sur les besoins des aînés, particulièrement les femmes. Selon lui, l'État a fait naitre un degré élevé de dépendance de la part des femmes âgées. Il est intéressant de noter que l'article de Snell fait ressortir davantage le besoin d'une forme de raisonnement axé sur une éthique publique exprimé par Clark. 
Pour terminer cet éditorial, j'aimerais souligner que les sciences humaines contribuent aux " Logos, " ou l'histoire du vieillissement, en fournissant un outil pour mettre à l'épreuve la justesse de nos approches systématiques envers la science et la pratique. Par justesse, j'entends le degré auquel nos méthodes scientifiques et notre formation technique correspondent aux expériences vécues par les personnes âgées, ainsi que le sens que ces personnes attribuent à cette expérience. (En plus des articles compris dans ce numéro, vous pouvez consulter un ouvrage publié en 1993 par Haldemann, portant sur les méthodes qualitatives en gérontologie.)

\section{Bibliographie}

Birren, J., Kenyon, G., Ruth, J.E., Schroots, J.J.F., \& Svensson, T. (Éds.). (à paraitre). Aging and Biography: Explorations in Adult Development. New York: Springer.

Cole, T., Achenbaum, A., Jakobi, P., \& Kastenbaum, R. (Éds.). (1993). Voices and Visions of Aging. New York: Springer.

Cole, T., Van Tassel, D., \& Kastenbaum, R. (Éds.) (1992). Handbook of Humanities and Aging. New York: Springer.

Haldemann, V., (Éd.). (1993). Qualitative Methods: Why? La Revue canadienne du vieillissement, numéro spécial, 12(2): 117-138.

Kenyon, G., Birren, J., \& Schroots, J.J.F. (Éds.). (1991). Metaphors of Aging in Science and the Humanities. New York: Springer.

Kenyon, G., \& Davidson, W. (1993). Ethics in An Aging Society. Dans Ethics and Aging, Writings in Gerontology, no 13. Ottawa: Conseil consultatif national sur le troisième âge.

Gary M. Kenyon, St. Thomas University 